\title{
ASO Author Reflections: Prognostic Role of Pathologic Complete Response After Neoadjuvant Therapy in Digestive Cancer
}

\author{
Tao Wan, MS ${ }^{1}$, and Yan-Ming Zhou, $\mathrm{MD}^{2}$ \\ ${ }^{1}$ Department of Gastrointestinal Surgery, Jiangxi Provincial People's Hospital, Nanchang, China; ${ }^{2}$ Department of \\ Hepatobiliary and Pancreatovascular Surgery, First Affiliated Hospital of Xiamen University, Xiamen, China
}

\section{PAST}

Some digestive cancer patients may achieve pathologic complete response (pCR) after neoadjuvant therapy (NAT). ${ }^{1}$ According to literature review, a retrospective cohort study ${ }^{2}$ showed that $\mathrm{pCR}$ was associated with improved overall survival (OS), whereas another study ${ }^{3}$ argued that OS did not differ significantly between pCR and non-pCR patients. Therefore, to date, the prognostic impact of pCR on survival in digestive cancers has not been completely established.

\section{PRESENT}

First, this study ${ }^{4}$ demonstrated that digestive cancer patients who achieved pCR after receiving NAT were more likely to have significantly better OS or disease-free survival (DFS). Second, molecular biology research on the group of patients with pCR should facilitate the finding of sensitive therapeutic targets in order to deliver tailored NAT to individual patients. Finally, pCR can be considered a valuable prognostic factor for better guidance in clinical decision making for postoperative adjuvant treatment and follow-up observation.

\footnotetext{
ASO Author Reflections offer a brief invited commentary on the article, The Prognostic Value of a Pathologic Complete Response After Neoadjuvant Therapy for Digestive Cancer: Systematic Review and Meta-Analysis of 21 Studies. Ann Surg Oncol. 2019;26:1412-20.
}

(C) Society of Surgical Oncology 2019

First Received: 16 April 2019;

Published Online: 22 April 2019

Y.-M. Zhou, MD

e-mail: zhouymsxy@sina.cn

\section{FUTURE}

Considering the great clinical implications of $\mathrm{pCR}$, the authors plan to explore possibilities for improving the pCR rate in the future, such as adding targeted agents in $\mathrm{NAT}^{5}$ and using concomitant boost radiotherapy as well as appropriate delay in the interval between NAT and surgery. ${ }^{6}$ It is anticipated that the clinical outcomes for digestive cancer patients will be further improved with continuous research progress in increasing the pCR rate.

DISCLOSURES There are no conflicts of interest.

\section{REFERENCES}

1. Cho H, Nakamura J, Asaumi Y, et al. Long-term survival outcomes of advanced gastric cancer patients who achieved a pathological complete response with neoadjuvant chemotherapy: a systematic review of the literature. Ann Surg Oncol. 2015;22:787-92.

2. Dossa F, Acuna SA, Rickles AS, et al. Association between adjuvant chemotherapy and overall survival in patients with rectal cancer and pathological complete response after neoadjuvant chemotherapy and resection. JAMA Oncol. 2018;4:930-7.

3. Mansour JC, Tang L, Shah M, et al. Does graded histologic response after neoadjuvant chemotherapy predict survival for completely resected gastric cancer? Ann Surg Oncol. 2007; 14:3412-8.

4. Wan T, Zhang XF, Liang $C$, et al. The prognostic value of a pathologic complete response after neoadjuvant therapy for digestive cancer: systematic review and meta-analysis of 21 studies. Ann Surg Oncol. 2019;26:1412-20.

5. Garcia M, Martinez-Villacampa M, Santos C, et al. Phase II study of preoperative bevacizumab, capecitabine, and radiotherapy for resectable locally advanced rectal cancer. BMC Cancer. 2015;15:59.

6. Tulchinsky H, Shmueli E, Figer A, Klausner JM, Rabau M. An interval $>7$ weeks between neoadjuvant therapy and surgery improves pathologic complete response and disease-free survival in patients with locally advanced rectal cancer. Ann Surg Oncol. 2008; 15:2661-7.

Publisher's Note Springer Nature remains neutral with regard to jurisdictional claims in published maps and institutional affiliations. 